\title{
THE COMPARISON OF IMMUNIZATION MECHANISMS AGAINST DISSEMINATING INFORMATION IN SOCIAL NETWORKS
}

\author{
Mohsen Raeisi ${ }^{1}$ and Behzad Akbari ${ }^{2}$ \\ ${ }^{1}$ Faculty of Computer and Information Technology Engineering, Qazvin Branch, Islamic \\ Azad University, Qazvin, Iran \\ ${ }^{2}$ Department of Computer Engineering, Tarbiat Modares University, Tehran, Iran
}

\begin{abstract}
These days considering expansion of networks, dissemination of information has become one of significant cases for researchers. In social networks in addition to social structures and people effectiveness on each other, Profit increase of sales, publishing a news or rumor, spread or diffusion of an idea can be mentioned. In social societies, people affect each other and with an individual's membership, his friends may join that group as well. In publishing a piece of news, independent of its nature there are different ways to expand it. Since information isn't always suitable and positive, this article is trying to introduce the immunization mechanism against this information. The meaning of immunization is a kind of Slow Publishing of such information in network. Therefor it has been tried in this article to slow down the publishing of information or even stop them. With comparison of presented methods for immunization and also presenting rate delay parameter, the immunization of methods were evaluated and we identified the most effective immunization method. Among existing methods for immunization and recommended methods, recommended methods also have an effective role in preventing spread of malicious rumor.
\end{abstract}

\section{KEYWORDS}

Social networks, information dissemination, immunization, delay rate

\section{INTRODUCTION}

The relationships among people often are organized in a network. For example, internet [1], web [2], social networks [2,3], are different kinds of these structures. Topology created by the relationships between people plays a major role in the dissemination of information [4]. One of dangers that always threat all networks is destructive information that by spreading in these networks can cause disruptions in them. Spread of types of noise in electricity networks [5], the virus spread in computer networks [6], the spread of disease in human networks [7], Harmful rumors spread on social networks are some examples of these destructions. Usually information which leads to destruction in network is called virus and also the preventing methods for them are called immunization mechanisms $[8,9]$.

Assuming the existence of a network and a model virus spread, immunization process in unofficial figure is equivalent to securing the minimum number of nodes in the network as causes stopping virus spread [10]. Immunization process is directly related to virus spread process. The simplest approach is fraction of nodes random immunization that is this number of nodes in the development of information is displayed ineffective. But in more complex methods, the role and importance of the nodes determination influences on the optimal strategies for their immunization [11]. 
Different models of information dissemination in a network are researched in different fields such as Computer science, sociology, physics and Epidemic. Two common kinds of them related to graph structure-based publishing and Independent model of the graph structure [12].

Considering the importance of this subject, this paper seeks to compare immunization mechanism based on network structure for presented information dissemination and study presented immunization mechanism against information dissemination in real and artificial networks.

\section{SPREAD OF RUMOR IMPORTANT FEATURES IN IMMUNIZATION}

Each node's features are effective in publishing rumor by that node, that we mention them and their role briefly.

1- Connections and density: the role of connections and density in network is that as the number of information (Number of links) is higher in network and so called the network is denser, the information publishes faster. Because there are more paths among users that any information choose that oath and go along to spread the rumor [13].

2- The degree of distribution: each node has input and output degree in network. If we assume that our networks are non-directional these two values will be the same. The degree of a node has an important role in dissemination and shows the number of one node's neighbors for dissemination [13, 14]. When we use PUSH protocol as the degree of one node is higher more information can be PUSH by it to its neighbors. Also based on riches and poos low, when one node is connected to a high node, get the information more than other nodes [15, 16].But it's noticeable that although one node is connected to the higher node, but considering presented rumor mechanism, when node $i$ receives a piece of information and according to $p_{\text {drop }}$ wants to spread it, choosing that special node is like $j$ that we want to get this information surely $p_{\text {Recievedata }}=\left(\frac{1}{\operatorname{deg} \text { ree }_{i}}\right)$, that as node degree is higher, the possibility of its information receive by node will decrease. But again it's noticeable that in this research the main goal is dissemination of information and coverage of the nodes by this notice not wanting a special node to receive information. So the degree is very important in dissemination.

3- Apical betweenness: is a criterion for measuring this subject that one node is located in how many short paths and as it's higher, represents that is in the shortest route, through these path choose its neighbors and spread rumor and because all paths are short the information will spread and delay rate of spread will get to the minimum.

4- The clustering coefficient: this criterion shows that the number of links rate among nodes neighbors are how many links are those. In another word, how many nodes neighbors are neighbors? This feature important because the height of this value in dissemination increase rumors redundancy of message and also prevents nodes isolation in networks [17].

\section{IMMUNIZATION STRATEGY}

In this research, research networks are evaluated by below methods:

1. Total degree immunization: as we mentioned before the degree of a node has a great role in dissemination so we will study the role of this feature. 
2. Betweenness immunization: based on the biggest betweenness of each node, immunize nodes not to participate in transfer, dissemination and receiving destructive information with virus.

3. Clustering coefficient immunization: high clustering coefficient nodes have important role in dissemination.

4. Immunization based on closeness: according to biggest value of proximity of the top of each node immunize nodes not to participate in transfer, dissemination and receiving destructive information with virus.

5. Eigenvalue immunization: nodes with the biggest eigenvalue have effective role in dissemination.

For example small world networks compared to Regular networks with the same number of nodes and links have high connectivity because of existence of shortcuts in these networks [18] in another word the small world networks have high clustering coefficient and virus disseminations happens faster in these kinds of networks.

\section{IMMUNIZATION MECHANISM}

Sometimes information may be Viruses, worms, Trojans [19] or harmful rumors [10, 20, 21] that the network is needed to be vaccinated or immunized against their dissemination.

Immunization mechanism acts in a way that first immunized nodes based on mentioned errors $10 \%, 20 \%, 30 \%, 40 \%$ and $50 \%$ (we considered here for these nodes $p_{p_{\text {drop }}}=100 \%$ ); means they delete each data which receive $100 \%$ and don't participate in its dissemination to other nodes. Then in next phase we will perform the protocol on immunized network and will record the dissemination rate for dissemination beginning. We continue this to fix the delay rate of dissemination in network.

\section{IMMUNIZATION NUMERICAL VALUES}

Here we perform immunization methods in 4 sample network and check that which network's features are more effective on immunization of that network. After immunizing the mechanism with different number of nodes for network and evaluating the rate of dissemination in each performance we got an average from delay rate and recorded it for that network and nodes in below diagrams. The used soft wares in this research are Matlab and Java. The reasons such as characteristics the structure of graph, node and dissemination protocol characteristics have crucial role in average delay rate [22].

In figure 1 we see a comparison of offered method of immunization in each Erdos-Renyi network. The results are:

- In this diagram it is observed that with immunization more than $30 \%$, the nodes with the highest degree ID goes to zero and network goes to immunization and face dramatic dissemination reduction.

- 2 other methods immunize the network with the spread of rumor in Erdos-Renyi network immunization are betweenness immunization and closeness. In these two methods when we immunize the network more than $30 \%$ with nodes of the highest betweenness and closeness, the delay rate to disseminating destructive rumor will go to the zero. 


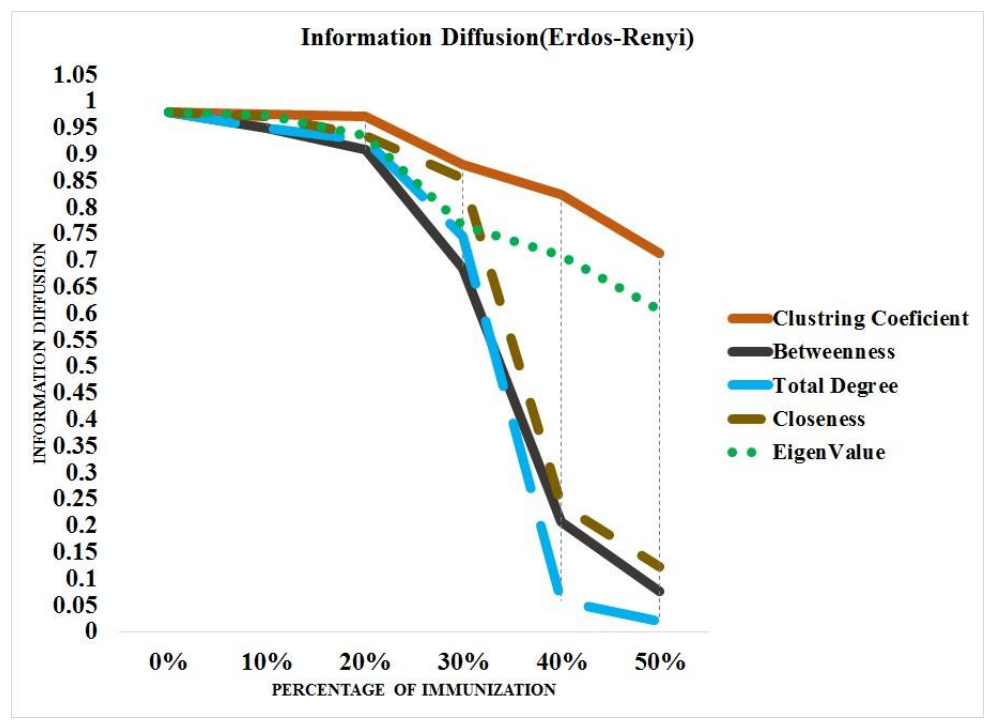

Figure 1: Comparison of all immunization methods in Erdos-Renyi network

- Clustering coefficient and eigenvalue methods are not suitable for this network because in more than $50 \%$ immunization it might be immunized.

After figure 1 that is immunization of Erdos-Renyi network with offered methods we conclude that the best method of immunization in this network is based on the biggest number of node degree and after that we can name two methods of betweenness and closeness in immunizing Erdos-Renyi network.

After that network is small world's turn. In figure 2 we observe the comparison of offered methods in this network. Analysis of diagram as follows:

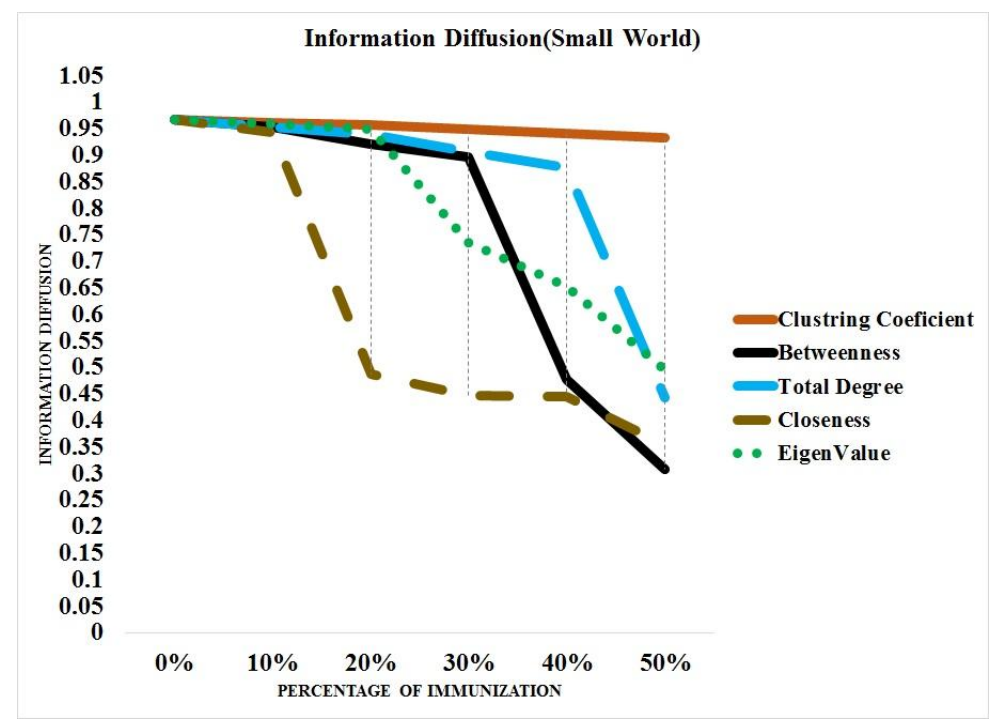

Figure 2: The comparison of offered methods for immunization in small world network

- Immunization based on closeness is one of effective methods in immunization of this network. In this mechanism between $10 \%$ and $20 \%$ removing nodes, ID changes from 
0.944 to 0.487 that it shows that immunizing nodes by this method is resistant against destructive rumor dissemination and slow down the dissemination.

- After closeness, betweenness after removing 30\% of nodes applies its effect and causes the dissemination speed reduction in small world network.

- In this network, eigenvalue method pass a right descending order and rumor dissemination get to 0.496 in $50 \%$ to 0.968 in $0 \%$.

- The most number of nodes also can't be more effective before $40 \%$ removing of nodes but after that it will be effective.

- Clustering coefficient in small world network is not suitable method because in more than $50 \%$ they may be immunized.

So we can conclude from figure 2 that all immunization offered methods except clustering coefficient for small world network were effective but betweenness and closeness have had more important role.

Scale free network is an artificial network that we checked in this research that we investigate immunization methods in this network to. In figure 3 we presented a comparison of immunization methods in scale free network. We will check the analysis of method briefly.

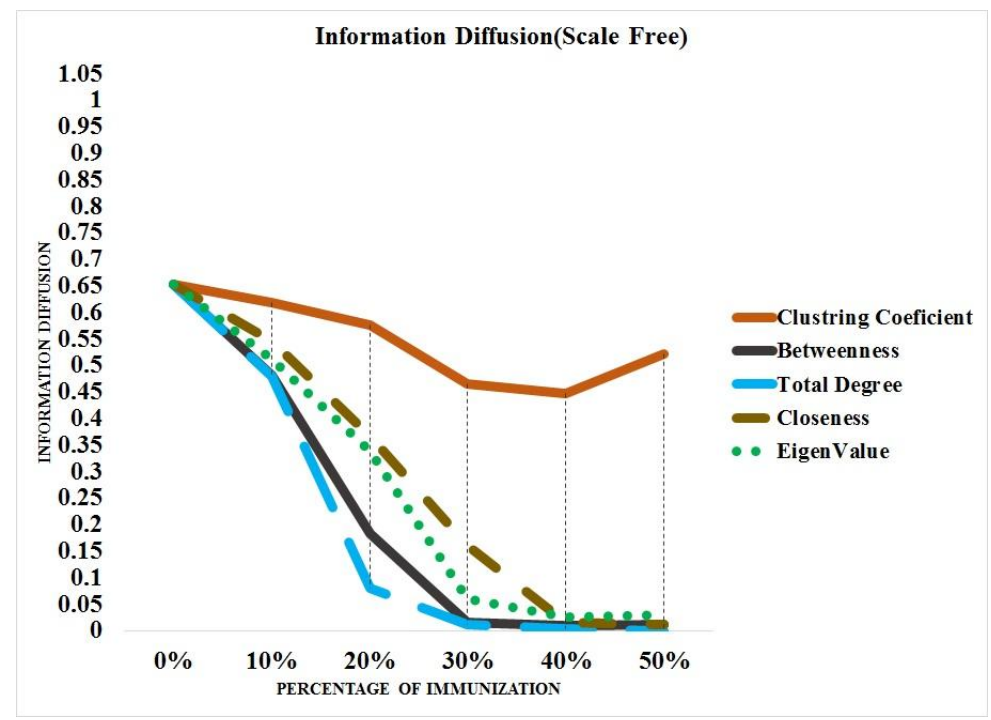

Figure 3: The comparison of offered methods of immunization in scale free network

- According to this network we see that immunization method based on degree causes the network goes to zero in more than $30 \%$ of nodes and in 50\% get the zero and network immunized $100 \%$. So this is the best method for immunization of this network.

- After this method the other ways which immunize scale free network are betweenness and closeness that is vaccinated with more than $30 \%$ immunization. This represents that as the delay rate of dissemination is lower it causes the news go faster from beginning node so if this node be immunized the possibility of destructive rumor dissemination will decrease dramatically.

- In next level eigenvalue method is effective. 
- Clustering coefficient method is not good method in scale free network because there is a possibility for immunization in more than $50 \%$. Another thing that is noticeable here is jumps from 40 to $50 \%$ removal of nodes. The reason of that is, with removing $40 \%$ of nodes the clustering coefficient will be zero so for removing the fifth $10 \%$ of nodes in network we should remove all $10 \%$ of primary nodes of network that are zero and because of that we will see this jumping in diagram.

According to figure 3 it can be concluded that immunization based on degree, betweenness, closeness and eigenvalue are the best methods for scale free network immunization.

After checking these 3 artificial networks it's time to also analyze immunization in a real network. The real network that we are going to check is Enron Email communication network. In figure 4 we can see this network with different immunization methods.

- The first methods that vaccinate network in more than $40 \%$ nodes immunization, are methods based on nodes degree and betweenness.

- Next two methods are eigenvalue and closeness.

- In the last part like Erdo-Renyi, small world and scale free networks there is clustering coefficient that is not good even in this real method.

Now we can according to real network collect these points:

Two methods of nodes degree and betweenness are the most important methods in this network immunization.

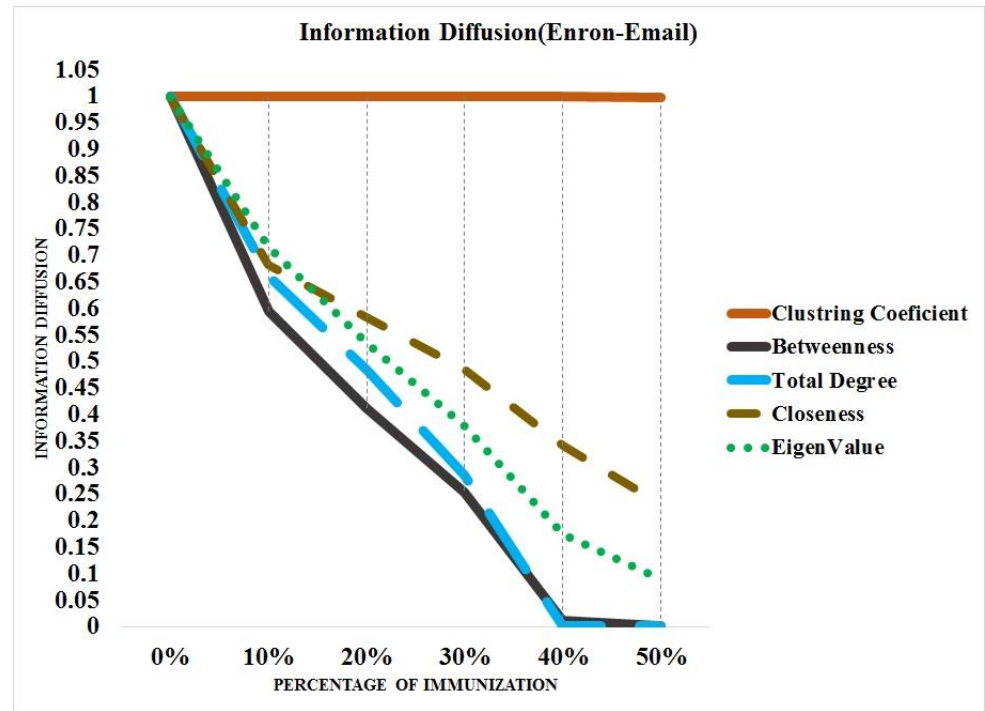

Figure 4: The comparison of offered methods of immunization in Enron Email communication network

After comparison of these 4 networks in immunization methods, we presented table 1 for introducing and comparing the best methods of immunization.

Studied networks Immunization mechanism: 
International Journal of Computer Networks \& Communications (IJCNC) Vol.7, No.4, July 2015

Table 1: View more effective method of immunization the network

\begin{tabular}{|c|c|c|c|c|c|}
\hline \multirow{2}{*}{ Studied networks } & \multicolumn{5}{|c|}{ Immunization mechanism } \\
\cline { 2 - 6 } & $\begin{array}{c}\text { Nodes } \\
\text { degree }\end{array}$ & Eigenvalue & Closeness & $\begin{array}{c}\text { Clustering } \\
\text { coefficient }\end{array}$ & Betweenness \\
\hline Erdos-Renyi & $\star$ & & & & $\star$ \\
\hline Small world & & & $\star$ & & $\star$ \\
\hline Scale free & $\star$ & & $\star$ & & $\star$ \\
\hline Enron Email & $\star$ & & & & \\
\hline
\end{tabular}

In table 1 we can conclude that in 3 networks the importance of the highest degree (ER, SF, EE), betweenness (EE, SF, SW) and closeness (SF, SW). In this way we can see the common factor of scale free network in these 3 immunization methods.

Now we want to compare networks on immunization systems and check based on immunization mechanism features, which networks have more important effects.

In figure 5 we can see the comparison of offered networks in immunization mechanism based on degree. The results of this diagram that we can get are as follows:

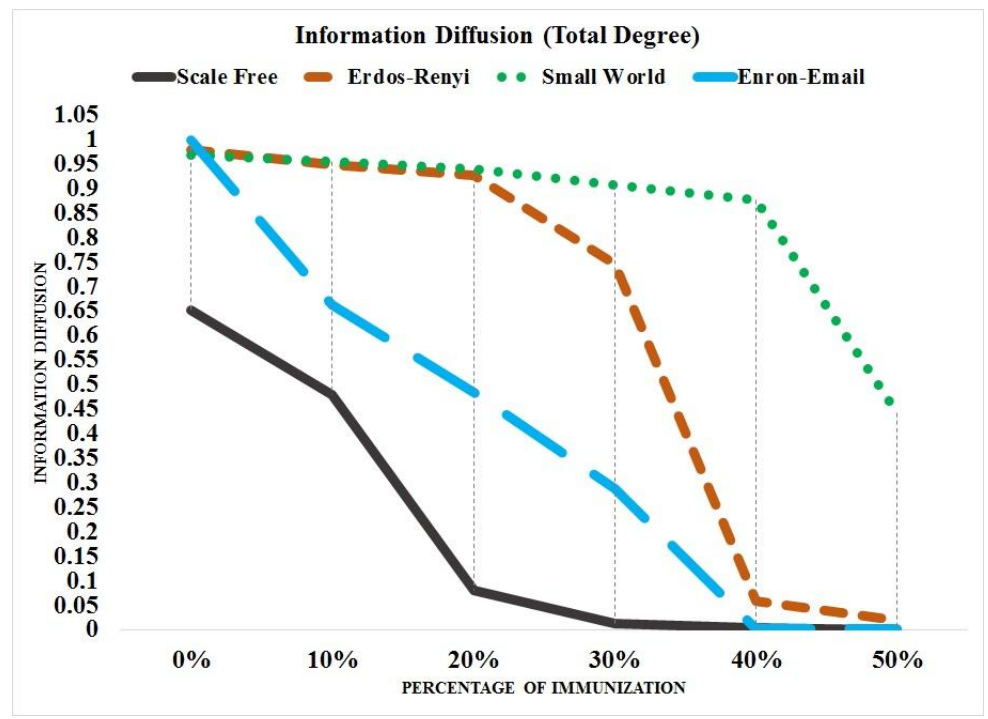

Figure 5: The comparison of offered networks in immunization mechanism based on degree

- Immunization based on the highest node degree method is such a method that in scale free and Enron networks shows the fastest reaction.

- According to this photo we can see that immunization based on degree in scale free network in more than $30 \%$ of nodes causes that network goes to zero and in $50 \%$ dissemination reach to zero and network get immunized $100 \%$. So this is the best method for this network.

- After scale free, Enron Email network after removing $40 \%$ of nodes goes to zero and dissemination will be slower and the network get vaccinate.

- In Erdos-Renyi we see that with immunization more than $30 \%$ of nodes that have the highest degree in network ID go to zero and the network goes through immunization and we face the significant reduction of dissemination. 
- The immunization of nodes degree before removing $40 \%$ of nodes cannot be effective on dissemination in small world network but after $40 \%$ we can see a tangible reduction.

So it can be said that immunization based on degree was effective for 3 study networks of scale free, Enron and Erdos-Renyi and it was more effective in scale free network.

After immunization mechanism based on degree it's the time of immunization based on betweenness. In figure 6 we can see the comparison of offered networks in this mechanism. Diagram analysis as follows:

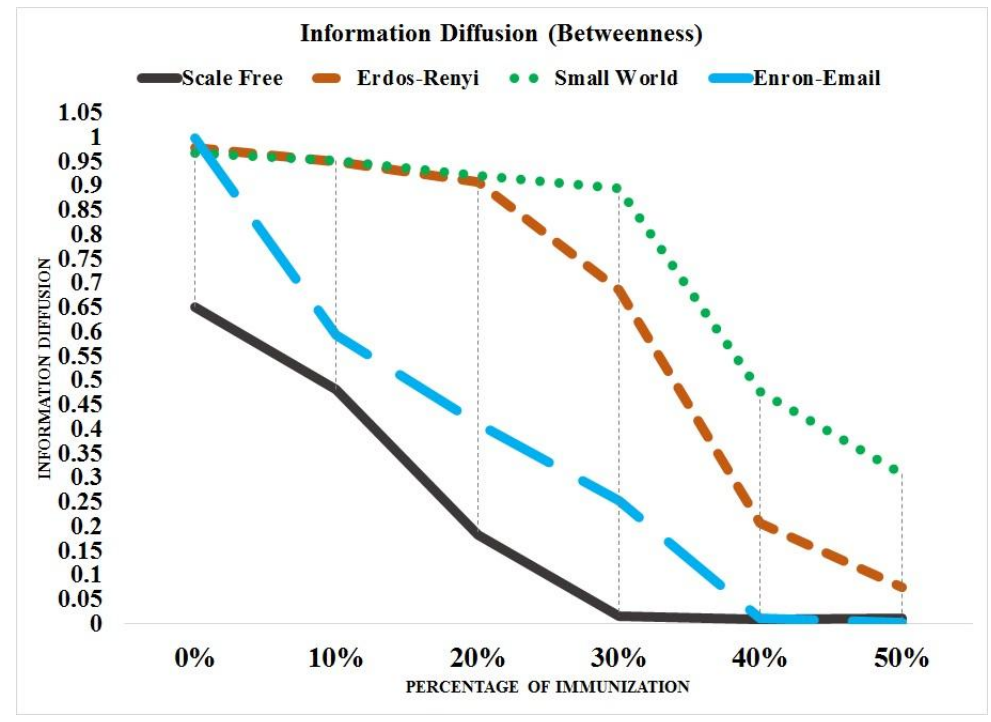

Figure 6: The comparison of offered networks in immunization mechanism of betweenness

- One of the methods that rapidly immunize the scale free network is betweenness that with immunization more than $30 \%$ the network get vaccinated. This represents that as delay rate of dissemination value is lower, it causes that the news spread faster from the beginning node so if this node is immunized then the dissemination of destructive rumor will be significantly reduced.

- The next network that will be immunized using this mechanism is Enron that after removing $40 \%$ of nodes with the highest value of betweenness even acts better than scale free and get to zero faster.

- The method in Erdos-Renyi that immunizes it with destructive rumor dissemination is betweenness. When we immunize this network with more than $30 \%$ of nodes that have the highest betweenness, dissemination rate for destructive rumor dissemination goes to zero.

- Betweenness mechanism after removing 30\% of nodes applies its influence in small world network and causes dissemination reduction in this network.

- So it can be concluded that immunization of betweenness for scale free and Enron is the best and causes that the mentioned network get vaccinated.

After betweenness is closeness immunization's turn. In figure 7 there is a comparison of offered networks in this mechanism. 


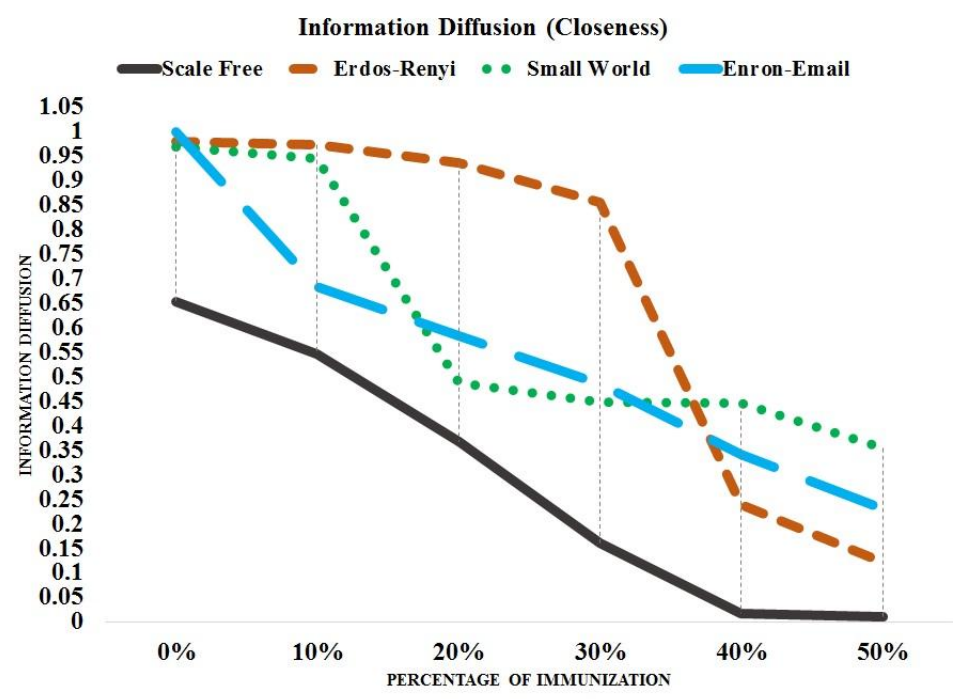

Figure 7: The comparison of offered networks in closeness mechanism

- As you can see in this figure, one of methods that immunizes scale free network rapidly is closeness.

- After this network, Erdos-Renyi has the best reaction and in this network when we immunize more than $30 \%$ of nodes that has more closeness, dissemination rate for destructive rumor dissemination will be zero.

- This method is effective in Enron too because reduces dissemination from 0.999 in $0 \%$ to 0.230 in $50 \%$ and causes the dissemination get slower and it's possible that with immunization more than $50 \%$ of node that has the highest closeness the network goes to zero.

- From the methods that has fastest reaction to nodes immunization in small world network is immunization based on the highest closeness. In this network between 10 to $20 \%$ removing of nodes ID changes from 0.944 to 0.487 that it represents that immunization with this method is resistant against destructive rumor and causes that dissemination get slower.

So we can conclude that this method is the best for scale free network and in terms of leveling has the best reaction in 10 to 20 percent of nodes immunization in small world network that will be faced with dissemination reduction.

After closeness we want to study eigenvalue method. In figure 8 there is a comparison of offered networks in this mechanism. 


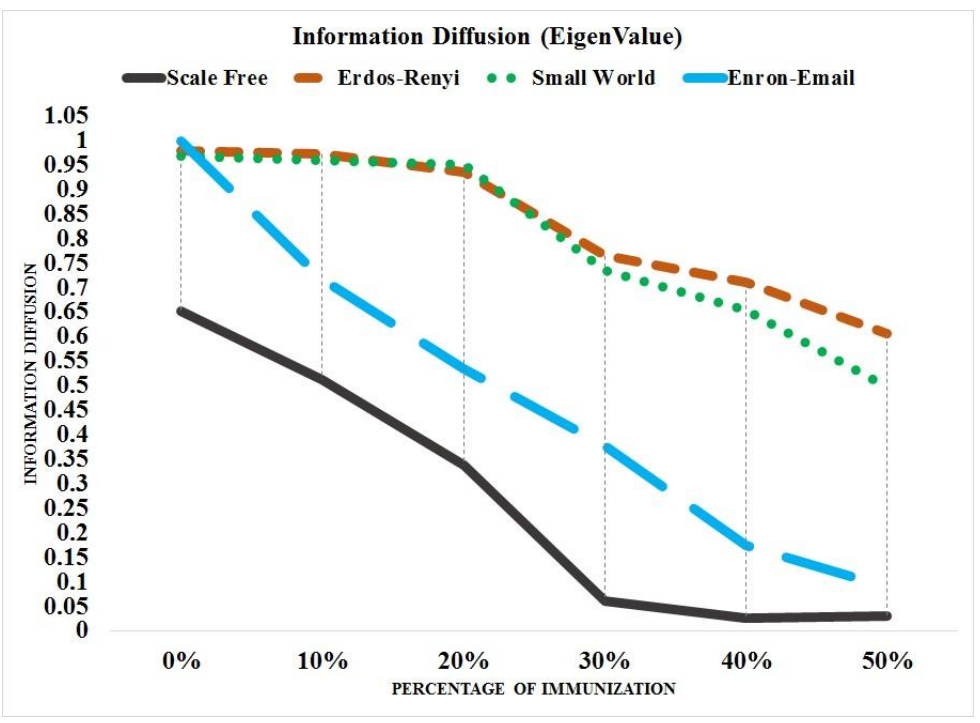

Figure 8: The comparison of offered networks in eigenvalue mechanism

- As you can see in this figure scale free network has the best reaction here too and causes dissemination reduction.

- After this network, we have Enron Email network that using eigenvalue mechanism can be vaccinated.

- This immunization method is not suitable for small world and Erdos-Renyi networks.

So we can conclude from picture 8 that scale free and Enron have the best reaction and scale free network even is better and small world and Erdos-Renyi networks are not suitable for this mechanism.

After immunization based on eigenvalue we will check clustering coefficient mechanism. In figure 9 there is a comparison of offered networks in this mechanism.

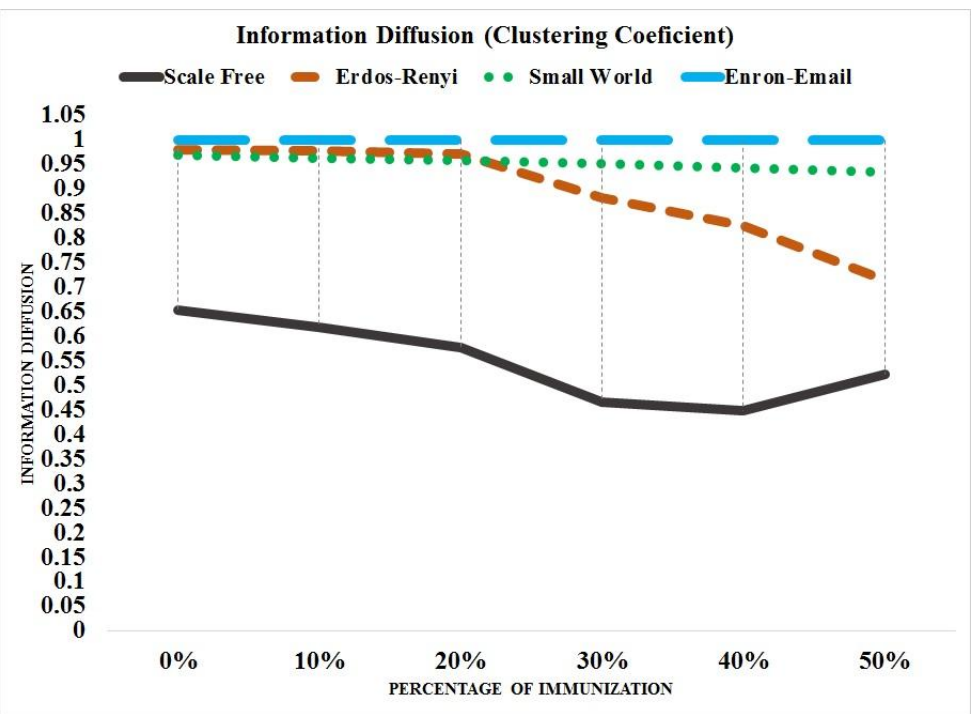

Figure 9: The comparison of offered networks in clustering coefficient mechanism for immunization. 
- As it is clear in this figure, clustering coefficient mechanism is not good for any of networks and is not also effective on these networks.

- The most reduction by this mechanism is related to Enron Email network that ID with 0.979 in $0 \%$ changes to 0.714 in $50 \%$.

- In Enron network we didn't see any changes with removing $50 \%$ of nodes with the highest clustering coefficient.

So we can conclude that clustering coefficient mechanism is not good for any of study networks at all.

\section{CONCLUSION}

Information dissemination without losing its generality among network users is always a challenging issue for researchers. Expand and spread of data is a fundamental process that occurs in the context of social networks. Presenting a mathematical analysis of discrete time, that with selecting the node exists between it the two links, checks the possibility of dissemination. The existence of one link between 2 nodes causes that if one of them has a prior piece of information, based on possibilities that node find its neighbor possibly and sent that information. The existence of one piece of news in social networks is important that this feature causes its fast expanding. That is the news that is prior must be disseminated in network. Now it is possible that on node (user) is not interested in its dissemination in network as a result removes that possibly and doesn't participate in its dissemination.

For evaluating the method, we used 4 networks that 3 of them were artificial and one was real. In rumor dissemination there is no interest always to this dissemination happen fast, but sometimes this piece of information that for its dissemination we used rumor protocol and rumor dissemination models, is a destructive rumor named virus. In front of this we have to act in a way that prevents its expanding or virus.

One of possible solutions for not disseminating of virus and destructive data is recognition of critical and important nodes.

These nodes recognition is possible based on node structure features. In this research we did by intentional and random methods the things that these nodes get immunized in rumor dissemination and not to expand the pollution in network then we compared them together for sample networks.

Finally by performing immunization mechanism on research networks we concluded that scale free network shows the best performance compared to other networks and clustering coefficient method is not suitable for any of them.

\section{REFERENCES}

[1] R. Pastor-Satorras, A. Vasquez, A. Vepignani, "Dynamical and correlation properties of the Internet," Physical Review Letters, Vol. 87, Issue 25, 2001.

[2] A. Mislove, k. P. Gummadi, P. Druschel, "Exploiting social network for internet search," Proceeding 5th Workshop on Hot Topics in Networks, Irvine, CA, USA, pp. 79-84, 2006.

[3] D. F. Nettleton, "Data mining of social networks represented as graphs," Computer Science Review, Vol. 7, Issue 1, pp. 1-34, 2013.

[4] M. Karsai, M. Kivela, R. K. Pan, K. Kaski, J. Kertesz, A. L. Barabasi, J. Saramaki, "Small but slow world: How network topology and burstiness slow down spreading," Physical Review E, Vol. 82, no. 2, 2011 . 
[5] E. Fee, D. M. Fox, AIDS: The making of chronic disease, 1st ed., University of California press, Berkeley, Los Angles, 1992.

[6] M. Cha, A. Mislove, K. P. Gummadi, "A measurement-driven analysis of information propagation in the flickr social networks," Proceeding of the 18th international conference on Word Wide Web, ACM, pp. 721-730, New York, USA, 2009.

[7] R. Pastor-Satorras, A. Vespignani, "Epidemic Spreading in Scale-Free Networks," Physical Review Letters, Vol. 86, no. 16, 2001.

[8] H. W. Hethcote, "The Mathematics of Infectious Diseases," SIAM Review, Vol. 42, no. 4, pp. 599653, 2000.

[9] C. L. Barrett, K. R. Bisset, S. G. Eubank, X. Feng, "EpiSimdemics: an efficient algorithm for simulating the spread of infectious disease over large realistic social networks," in Proceedings of the 2008 ACM/IEEE Conference on Supercomputing, Vol. 37, pp. 1-12, USA, 2008.

[10] J. O. Kephart, S. R. White, "Measuring and modeling computer virus prevalence," In IEEE Symposium on Research in Security and Privacy, pp. 2-15, Oakland, USA, 1993.

[11] R. Pastor-Satorras, A. Vespignani, "Immunization of complex networks," Physical Review E, Vol. 65, Issue 3, 2002.

[12] Ch. M. Kribs-zaleta, J. X. Velasco-Hernandez, "A simple vaccination model with multiple endemic states," Mathematical biosciences, Vol 164, Issue 2, pp 183-201, 2000.

[13] R. Bakhshi, Gossiping Models, Ph.D. Thesis, Computing Science Department, Vrije University, 2011.

[14] G. Frederick, Gossip Algorithms, Florida Institute of Technology Technical Report, no. CSE 5400, 2008.

[15] P. T. Eugster, R. Guerraoui, A. Kermarrec, L. Massoulieacute, "Epidemic Information Dissemination in Distributed Systems," Computer, Vol. 37, no. 5, pp 60-67, 2004.

[16] M. Starnini, A. Machens, C. Cattuto, A. Barrat, R. P. Satorras, "Immunization strategies for epidemic processes in time-varying contact networks," Journal of Theoretical Biology, Vol. 327, pp.89-100, 2013.

[17] M. E. J. Newman, “The Structure and Function of Complex Networks," SIAM Rev, Vol 45, 2003.

[18] R. Albert, H. Jeong, A. L. Barabasi, "Error and attack tolerance of complex networks," Nature, Vol. 46, no. 6794, pp. 378-382, 2000.

[19] D. shah, T. R. Zaman, "Rumors in a network: who's the culprit," Transaction on information Theory, Vol. 57, no. 8, pp. 5163-5181, 2011.

[20] G. Giakkoupis, A. Gionis, E. Terzi, P. Tsaparas, "Models and Algorithms for Network Immunization," Journal of More presentations by epidemic r, 2014.

[21] P. G. Lind, L. R. da Silva, J. S. Andrade, H. J. Herrmann, "Spreading gossip in social networks," Physicial Review E, Vol. 76, 2007.

[22] Y. Xia, D. J. Hill, “Attack vulnerability of complex communication,” IEEE Transaction Circuits Syst. II, Exp. Briefs, Vol. 55, no. 1, pp. 65-69, 2008.

\section{AUTHORS}

Mohsen Raeisi received his B.Sc. degree in computer engineering from Bandar Abbas University in Iran in 2009. He pursued his education and got his M.Sc. degree in information technology engineering orientation of computer networks from Qazvin University in Iran in 2015. He studied Information Diffusion in Social Networks based on rumor spreading and prevention approach as his M.Sc. thesis project. His research interests are complex and social Networks. He is now administrators of computer networks in the province hormozgan gas company in iran.

Behzad Akbari received the B.Sc., M.Sc., and Ph.D. degrees in Computer Engineering from Iran Sharif University of Technology in 1999, 2002 and 2008, respectively. He is currently an assistant professor in the Department of Electrical and Computer Engineering, Tarbiat Modares University, Tehran, Iran. His research interests are performance modelling/evaluation, Interconnection networks, Computer networks, Network security, Internet engineering and Multimedia systems.
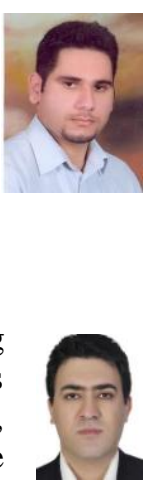